\title{
The significance of angiotensin II type 1 receptor (AT1 receptor) in renal transplant injury
}

\author{
Agnieszka Sas-Strózik ${ }^{A-F}$, Magdalena Krajewska ${ }^{\mathrm{E}, \mathrm{F}}$, Mirosław Banasik ${ }^{\mathrm{A}-\mathrm{F}}$ \\ Department of Nephrology and Transplantation Medicine, Wroclaw Medical University, Poland \\ A - research concept and design; $B$ - collection and/or assembly of data; $C$ - data analysis and interpretation; \\ $D$ - writing the article; $E$ - critical revision of the article; $F$ - final approval of the article
}

Address for correspondence

Agnieszka Sas-Strózik

E-mail:a_sas@op.pl

\section{Funding sources}

The research was financially supported by the Ministry of Health subvention according to number STM.C160.20.115 from the IT Simple system of Wroclaw Medical University.

Conflict of interest

None declared

Received on March 19, 2020

Reviewed on April 25, 2020

Accepted on April 29, 2020

Published online on May 27, 2020

\begin{abstract}
Humoral response beyond human leukocyte antigen (HLA) is of great interest in the transplant community. We decided to summarize the data on a new antigenic target called angiotensin II type 1 receptor (AT1 receptor). Non-HLA antibodies can now be detected in routine clinical care of patients after transplantation, but their role is not fully understood. Numerous analyses showed that non-HLA response may exert a higher risk of allograft rejection and allograft loss independently of the HLA system. Non-HLA response may even have a higher rate of antibody-mediated rejection. Information regarding antigen target, as well as the pathophysiology of its antibodies and diagnostic tools, is essential for a better understanding of non-HLA humoral response. Angiotensin II type 1 receptors are the most recognized target for non-HLA antibodies. Anti-ATIR Abs (anti-angiotensin II type 1-receptor-activating antibodies) may identify renal transplant patients at higher risk of graft rejection and loss. The presence of AT1 receptor expression analyzed together with anti-AT1R Abs should be considered for better transplant immunological risk assessment. Further assessment is required for a better understanding and to create appropriate therapeutic strategies.
\end{abstract}

Key words: angiotensin AT1 receptor, non-HLA antibodies, anti-AT1R Abs, antibody-mediated rejection, renal transplantation

Cite as

Sas-Strózik A, Krajewska M, Banasik M. The significance of angiotensin II type 1 receptor (AT1 receptor) in renal transplant injury. Adv Clin Exp Med. 2020;29(5):629-633. doi:10.17219/acem/121510

DOI

10.17219/acem/121510

\section{Copyright}

Copyright by Author(s)

This is an article distributed under the terms of the

Creative Commons Attribution 3.0 Unported (CC BY 3.0)

(https://creativecommons.org/licenses/by/3.0/) 


\section{Introduction}

Antibody-mediated rejection (AMR) is currently the main cause of graft loss. ${ }^{1}$ The influence of anti-HLA antibodies on transplant injury is well known and described as humoral theory of transplantation. ${ }^{2-4}$ Recently, non-HLA antibodies have been more often considered an additional factor which may have a negative impact on the transplant. ${ }^{5}$ The search for the role of these antibodies in renal transplant rejection has become more vital and has led to the discovery of many specific targets of their binding, other than HLA. Non-HLA antigens may be expressed on endothelial and epithelial cells but also parenchymal cells and circulating immune cells, which may be objectives for non-HLA antibodies. Among them, antibodies directed against angiotensin II type 1 receptor (anti-AT1R Abs) seem to be more important and should be considered a potential cause of transplant injury. AntiAT1R Abs were described as associated with allograft rejection. ${ }^{6-8}$ It seems possible that circulating anti-AT1R Abs might indicate renal transplant recipients at an increased risk of graft rejection and loss who are not discovered by the HLA system.

In this review, we discussed the significance of AT1 receptor as a potential target for anti-AT1R Abs. We also analyzed the pathomechanism of transplant injury and clinical consequences of the damage. Finally, we considered potential diagnostic implementation and therapeutic implications.

\section{AT1 receptor as a potential target for non-HLA antibodies}

The distribution of AT1 receptors occurs in many organs and, after activation by angiotensin II, has an impact on the vasoconstriction of vascular smooth muscle cells, sodium reabsorption in proximal tubules and aldosterone secretion in the adrenal cortex. ${ }^{9,10}$

Angiotensin II type 1 receptor (AT1 receptor) is G protein-coupled receptor that contains 7 transmembrane loops with the antibody binding place located on the second loop. ${ }^{11}$ It is activated by angiotensin II, which mediates some physiological and pathophysiologic processes with arterial blood pressure or water-salt balance. ${ }^{12}$ The human gene for AT1 receptor is located on chromosome 3 and contains 4 exons. ${ }^{13}$ There are some polymorphisms of AT1 receptors but the most known is $\mathrm{A} 1166 \mathrm{C}$, which is connected with increased sensitivity to angiotensin II and cardiovascular and renal insufficiency. ${ }^{14}$ The stimulation of AT1 receptors by anti-AT1R Abs involves the activation of nuclear factor- $\kappa \beta$ and causes inflammatory response. ${ }^{15}$ Stimulation of higher receptors has also been revealed due to cardiovascular complications connected with remodeling in heart, kidney and vessels, and caused increased mortality and morbidity. ${ }^{16}$
Many studies have shown that the significant presence of anti-AT1R Abs before transplantation is an important risk factor of graft loss. ${ }^{7,17}$ Some studies have also revealed a potential influence of a higher level of anti-AT1R Abs on graft failure and worse functioning of renal transplant. ${ }^{6,18}$ In their study, Dragun at al. presented 16 allograft recipients with refractory rejection and malignant hypertension and anti-AT1R Abs but without donor HLAspecific antibodies (HLA-DSAs). ${ }^{15}$

These findings challenged us to analyze the presence of AT1 receptors in renal transplant biopsies. ${ }^{19}$ As far as we know, nobody has analyzed AT1 receptors in renal transplant biopsies for cause (deterioration in function or proteinuria), so this research was pioneering. We detected positive immunostaining of AT1 receptors in tubular epithelium in $26.3 \%$ (42/118) of patients who had indication biopsy. The expression was estimated based on a three-step scale described as lack of expression (0), low immunoreactivity (1) and high expression (2). The expression was assessed as high in 7 patients and as low in 35 patients. What seems to be of high clinical significance is that one-year post-biopsy graft loss in patients with AT1 receptors expression in tubules was significantly higher compared to patients without such expression. ${ }^{19}$

The analysis of 156 renal transplant patients for the expression of the AT1 receptor and, additionally, the presence of anti-AT1R Abs showed that AT1 receptors are also detected in other renal compartments. ${ }^{20}$ In this group, 6 patients had positive expression in microcirculation (glomeruli and peritubular capillaries), which was connected with antibody-mediated rejection and high graft loss.

\section{Pathomechanism of injury}

Non-HLA antibodies, like anti-AT1R Abs, can be present before transplantation or occur de novo after transplantation. ${ }^{21}$ Many papers have revealed that when HLA antibodies and non-HLA antibodies occur together, they can cause much shorter graft survival compared to the situation when only 1 group of these antibodies is present. ${ }^{7}$ This is attributed to their synergistic impact on the structure of the endothelium. It is assumed that HLA antibodies cause endothelial damage and, as a result, autoantigens are exposed, which can lead to the formation of autoantibodies. ${ }^{22}$ On the other hand, non-HLA antibodies may provoke an inflammatory response and this process can cause upregulation of HLA expression; in consequence, the graft graft becomes more prone to alloimmune response. ${ }^{23}$

Angiotensin II type 1 receptor is prevalent on vascular endothelium and performs many significant functions. For example, it stimulates angiotensin II both in physiological and pathophysiological ways. ${ }^{12}$ It is involved in vasoconstriction, blood pressure regulation, and water and salt balance. It is the main mediator of oxidative stress and reduced activity of nitric oxide (NO). Angiotensin II causes 
endothelial dysfunction and stimulates pro-inflammation in vascular smooth muscle cells. ${ }^{24}$ It is thought that an increased level of angiotensin II may lead to acute ischemic complications. It can stimulate local metalloproteinases and, in the end, provoke high possibility of rupture. ${ }^{25}$

Stimulation of AT1 receptors by autoantibodies induces phosphorylation of the ERK1/2 pathway in the endothelium. It raises the binding activity of transcription factor nuclear factor- $\mathrm{k}$ and activator-protein 1 . This process may result in the synthesis of proteins, cell migration, inflammation and fibrosis. ${ }^{26,27}$ Dragun et al. revealed that only IgG1 and IgG3 subclasses can cause this type of agonistic effect. ${ }^{15}$

Due to their noticeable role in many molecular processes, anti-AT1R Abs are considered to be a significant risk factor in vascular diseases such as pre-eclampsia, systemic sclerosis and malignant hypertension. ${ }^{28,29}$

\section{The clinical significance of AT1 receptors in renal transplantation}

The first report about the impact of antibodies against angiotensin II type-1 receptor (anti-AT1R Abs) appeared in a study by Dragun et al., which indicated the influence of these antibodies on the kidney antibody-mediated rejection, where the presence of anti-AT1R Abs with the absence of anti-HLA was detected in 16 patients with progressive graft failure after renal transplantation. ${ }^{15}$

The presence of anti-AT1R Abs before the transplantation is a separate risk factor for graft failure both shortly after transplantation and in later outcome. ${ }^{30}$ Many studies have shown that patients with the anti-AT1R Abs (+) had a worse graft function are more frequent graft loss than the groups of patients where anti-AT1R Abs were absent. ${ }^{6,31}$

Giral et al. in their research focused on the presensitization against AT1 receptors and its impact on graft survival and the risk of acute rejection. ${ }^{7}$ The study involved 599 patients who underwent renal transplantation. A higher level of anti-AT1R Abs was identified in 283 patients (47.2\%) before renal transplantation. The study assumed an elevated level of anti-AT1R Abs as $>10 \mathrm{U} / \mathrm{L}$. In the risk group, where anti-AT1R Abs were positive, the probability of graft failure was 2.6-fold higher 3 years after transplantation and 1.9-fold higher risk of acute rejection during the first 4 months after transplantation. Therefore, a high level of anti-AT1R Abs should be considered a significant risk factor for rejection.

The occurrence of anti-AT1R Abs in renal transplant recipients seems to greatly affect graft survival and is an additional factor of graft rejection in patients with a higher immunological risk.

Antibodies against AT1 receptors may develop in many pathways. The transplant process itself can cause increased expression of AT1 receptors; ischemia and reperfusion injury by oxidative stress may also lead to an immune response. ${ }^{23}$

The pre-transplant presence of anti-AT1R Abs may be significant in predicting anti-AT1R Ab-related rejection. ${ }^{34}$ The evaluation of antibody-medicated rejection (AMR) risk based solely on the HLA pre-sensitization seems to be inadequate. In a study by Taniguchi et al., 351 patients after renal transplantation were taken into consideration. The patients were divided into a group with abnormal biopsies $(\mathrm{n}=134)$ and a control group $(\mathrm{n}=217){ }^{6}$ Anti-AT1R Abs were detected using quantitative enzymelinked immunosorbent assay (ELISA) test. A positive level of those antibodies was assumed to be $15-25 \mathrm{U} / \mathrm{mL}$ and a negative one $<10 \mathrm{U} / \mathrm{mL}$. Of all the patients, $17 \%(\mathrm{n}=60)$ had a positive level of anti-AT1R Abs. Thirty-five of those patients were anti-AT1R Abs-positive only at the time before transplantation and 1 patient from this group lost his graft. However, the remaining 25 patients, who were anti-AT1R Abs-positive, both before and after transplantation, had significantly greater graft loss. In the group of patients $(n=11)$ in which anti-AT1R Abs occurred de novo, the graft loss was observed in $64 \%$ of the patients $(\mathrm{n}=7)$. In the group of patients with abnormal biopsies, $14 \%(19 / 134)$ of patients were pre-transplant positive and $84 \%(16 / 19)$ of them stayed positive also after transplantation. In the control group, $19 \%$ of the patients (41/217) were anti-AT1R Abs-positive before transplantation, but only $22 \%(9 / 41)$ of them maintained the positive level of those antibodies after transplantation. Graft outcome revealed that $79 \%(19 / 24)$ of the group of patients with abnormal biopsies with post-transplant anti-AT1R Abspositive had lost their grafts, whereas in the control group, there were no cases of graft loss $(p<0.001)$. Another factor investigated in this study was the influence of antiAT1R Abs on graft survival with the presence or absence of donor-specific antibodies (DSA). It was observed that the worst graft survival was in the group of patients who presented both post-transplant anti-AT1R Abs and DSA $(p=0.007)$. The study concluded that despite the role of HLA antibodies on graft failure, non-HLA antibodies like anti-AT1R Abs also have a significant influence on graft function deterioration and, finally, rejection.

In the analysis of 117 patients after renal transplantation, arteritis in renal biopsy was significantly more frequent in anti-AT1R Abs $(+)$ group $(3 / 27,11.1 \%)$ than in anti-AT1R Abs $(-)$ group $(1 / 90,1.1 \%)(p=0.038)$. The study showed that the pre-transplant presence of anti-AT1R Abs can influence the graft function as an independent risk factor, which can result in earlier graft failure, rejection and graft loss. ${ }^{8}$

Our very last analysis of the expression of AT1 receptor together with its antibodies in patients who had a renal transplant indication biopsy showed that the presence of anti-AT1R Abs in serum together with the expression of AT1 receptor in transplant biopsy was associated with a significantly higher graft los. ${ }^{20}$ 
Anti-AT1R Abs may possibly identify renal transplant recipients at high risk of allograft rejection and loss independently of the HLA system. In the analysis of 1,845 renal transplant recipients, donor-specific HLA antibodies (DSA) and anti-AT1R Abs were measured at the time of the first acute rejection episode or 1 year after transplantation. ${ }^{32}$ Transplant biopsy was performed to assess the rejection phenotype and endothelial activation. The analysis showed that 371 (20.1\%) participants had anti-AT1R Abs, 334 (18.1\%) had DSA and 133 (7.2\%) had both.

Additionally, patients with anti-AT1R Abs had a higher rate of antibody-mediated rejection compared to participants without anti-AT1R Abs. Among 77 renal transplant recipients with histological features of AMR but without DSAs, 51 (66.2\%) had anti-AT1R Abs. What is more, antiAT1R Abs-associated rejection was marked by a higher level of endothelial-associated transcripts and lack of complement deposition in allograft capillaries.

Monitoring of non-HLA anti-AT1R Abs, in addition to the current immunologic assessment of renal transplant recipients, may have clinical significance in graft protection. Understanding complement-independent antiAT1R Abs-mediated rejection may help to create a new therapeutic approach targeting circulating anti-ATR Abs to improve allograft survival. Nowadays, the therapeutic strategy in recipients with antibody-mediated rejection involves removing circulating DSA, blocking the DSA effects, and reducing the production of antibodies. ${ }^{33}$ The identification of anti-AT1R Abs may significantly affect the clinical routine care of transplant recipients, as it may facilitate the development of therapeutic strategies. Further research should evaluate the usefulness of current therapies, such as plasma exchange and IVIG, which are standard care in the therapy of patients with anti-HLA AMR. Potential therapeutic strategies may include a selective blockade of AT1 receptors using sartans..$^{5,32,34,35}$ However, no clinical trial has been presented to prove the potential benefit of such approaches.

\section{Conclusions}

Angiotensin II type 1 receptors are an important target for non-HLA antibodies, which may cause injury of renal transplant. Independently of the HLA system, monitoring of anti-AT1R Abs may recognize renal transplant patients who are at a higher risk of graft rejection and loss. The presence of AT1 receptor expression in renal biopsy analyzed together with anti-AT1R Abs should be considered for better transplant immunological risk assessment.

\section{ORCID iDs}

Agnieszka Sas-Strózik (D) https://orcid.org/0000-0002-1068-0893 Magdalena Krajewska (D) https://orcid.org/0000-0002-2632-2409 Mirosław Banasik (D) https://orcid.org/0000-0002-0588-1551

\section{References}

1. Sellares J, de Freitas DG, Mengel M, et al. Understanding the causes of kidney transplant failure: The dominant role of antibody-mediated rejection and nonadherence. Am J Transplant. 2012;12(2):388-399.

2. Terasaki PI. Humoral theory of transplantation. Am J Transplant. 2003; 3(6):665-673.

3. Cai J, Terasaki PI. Humoral theory of transplantation: Mechanism, prevention, and treatment. Hum Immunol. 2005;66(4):334-342.

4. Terasaki PI, Cai J. Humoral theory of transplantation: Further evidence. Curr Opin Immunol. 2005;17(5):541-545.

5. Dragun D, Philippe A, Catar R. Role of non-HLA antibodies in organ transplantation. Curr Opin Organ Transplant. 2012;17(4):440-445.

6. Taniguchi M, Rebellato LM, Cai J, et al. Higher risk of kidney graft failure in the presence of anti-angiotensin II type-1 receptor antibodies. Am J Transplant. 2013;13(10):2577-2589.

7. Giral M, Foucher Y, Dufay A, et al. Pretransplant sensitization against angiotensin II type 1 receptor is a risk factor for acute rejection and graft loss. Am J Transplant. 2013;13(10):2567-2576.

8. Banasik M, Boratynska M, Koscielska-Kasprzak K, et al. The influence of non-HLA antibodies directed against angiotensin II type 1 receptor (AT1R) on early renal transplant outcomes. Transpl Int. 2014;27(10): 1029-1038.

9. Gasc JM, Shanmugam S, Sibony M, Corvol P. Tissue-specific expression of type 1 angiotensin II receptor subtypes. An in situ hybridization study. Hypertension. 1994;24(5):531-537.

10. Gurley SB, Riquier-Brison ADM, Schnermann J, et al. AT1A angiotensin receptors in the renal proximal tubule regulate blood pressure. Cell Metab. 2011;13(4):469-475.

11. Reinsmoen $\mathrm{NL}$, Lai $\mathrm{CH}$, Heidecke $\mathrm{H}$, et al. Anti-angiotensin type 1 receptor antibodies associated with antibody-mediated rejection in donor HLA antibody negative patients. Transplantation. 2010; 90(12):1473-1477.

12. Hunyady L, Catt KJ. Pleiotropic AT1 receptor signaling pathways mediating physiological and pathogenic actions of angiotensin II. Mol Endocrinol. 2006;20(5):953-970.

13. Dragun D. Humoral responses directed against non-human leukocyte antigens in solid-organ transplantation. Transplantation. 2008; 86(8):1019-1025.

14. Miller JA, Scholey JW. The impact of renin-angiotensin system polymorphisms on physiological and pathophysiological processes in humans. Curr Opin Nephrol. 2004;13(1):101-106.

15. Dragun D, Muller DN, Brasen JH, et al. Angiotensin II type 1-receptor activating antibodies in renal-allograft rejection. NEJM. 2005;352(6): 558-569.

16. Dzau VJ. Theodore Cooper Lecture: Tissue angiotensin and pathobiology of vascular disease: A unifying hypothesis. Hypertension. 2001;37(4):1047-1052.

17. Banasik M, Boratynska M, Koscielska-Kasprzak K, et al. Non-HLA antibodies: Angiotensin II type 1 receptor (anti-AT1R) and endothelin-1 type A receptor (anti-ETAR) are associated with renal allograft injury and graft loss. Transplant Proc. 2014;46(8):2618-2621.

18. Banasik M, Boratynska M, Koscielska-Kasprzak K, et al. The impact of de novo donor-specific anti-human leukocyte antigen antibodies on 5-year renal transplant outcome. Transplant Proc. 2013;45(4): 1449-1452.

19. Sas A, Donizy P, Koscielska-Kasprzak K, et al. Histopathological relevance of angiotensin II type 1 receptor in renal transplant biopsy. Transplant Proc. 2018;50(6):1847-1849.

20. Sas-Strózik A, Donizy P, Koscielska-Kasprzak K, et al. Angiotensin II type 1 receptor (AT1R) expression in renal transplant biopsies and anti-AT1R antibodies in serum indicates the risk of transplant loss. Transplant Proc. https://doi.org/10.1016/j.transproceed.2020.01.126

21. Michielsen LA, van Zuilen AD, Krebber MM, Verhaar MC, Otten HG. Clinical value of non-HLA antibodies in kidney transplantation: Still an enigma? Transplantation Rev. 2016;30(4):195-202.

22. Wang S, Zhang C, Wang J, et al. Endothelial cells in antibody-mediated rejection of kidney transplantation: Pathogenesis mechanisms and therapeutic implications. J Immunol Res. 2017;2017:8746303.

23. Reinsmoen NL. Role of angiotensin II type 1 receptor-activating antibodies in solid organ transplantation. Hum Immunol. 2013;74(11): 1474-1477. 
24. Kranzhofer R, Schmidt J, Pfeiffer CA, Hagl S, Libby P, Kubler W. Angiotensin induces inflammatory activation of human vascular smooth muscle cells. Arterioscler Thromb Vasc Biol. 1999;19(7):1623-1629.

25. Rouet-Benzineb P, Gontero B, Dreyfus P, Lafuma C. Angiotensin II induces nuclear factor - kappa B activation in cultured neonatal rat cardiomyocytes through protein kinase $\mathrm{C}$ signaling pathway. $\mathrm{J} \mathrm{Mol}$ Cell Cardiol. 2000;32(10):1767-1778.

26. Oliveira L, Costa-Neto CM, Nakaie CR, Schreier S, Shimuta SI, Paiva AC. The angiotensin II AT1 receptor structure-activity correlations in the light of rhodopsin structure. Physiol Rev. 2007;87(2):565-592.

27. Zhang H, Unal H, Gati C, et al. Structure of the angiotensin receptor revealed by serial femtosecond crystallography. Cell. 2015;161(4): 833-844.

28. Xia Y, Kellems RE. Angiotensin receptor agonistic autoantibodies and hypertension: Preeclampsia and beyond. Circ Res. 2013;113(1):78-87.

29. Crowley SD, Gurley SB, Herrera MJ, et al. Angiotensin II causes hypertension and cardiac hypertrophy through its receptors in the kidney. PNAS. 2006;103(47):17985-17990.

30. Banasik M, Boratynska M, Koscielska-Kasprzak K, et al. Long-term follow-up of non-HLA and anti-HLA antibodies: Incidence and importance in renal transplantation. Transplant Proc. 2013;45(4):1462-1465.
31. Fuss A, Hope CM, Deayton S, et al. C4d-negative antibody-mediated rejection with high anti-angiotensin II type I receptor antibodies in absence of donor-specific antibodies. Nephrology (Carlton). 2015;20(7):467-473.

32. Lefaucheur C, Viglietti D, Bouatou Y, et al. Non-HLA agonistic antiangiotensin II type 1 receptor antibodies induce a distinctive phenotype of antibody-mediated rejection in kidney transplant recipients. Kidney Int. 2019;96(1):189-201.

33. Velidedeoglu E, Cavaillé-Coll MW, Bala S, Belen OA, Wang Y, Albrecht R. Summary of 2017 FDA Public Workshop: Antibody-mediated rejection in kidney transplantation. Transplantation. 2018;102(6): e257-e264. doi:10.1097/TP.0000000000002141

34. Carroll RP, Riceman M, Hope CM, et al. Angiotensin II type-1 receptor antibody (AT1Rab) associated humoral rejection and the effect of peri operative plasma exchange and candesartan. Hum Immunol. 2016;77(12):1154-1158

35. Weidanz JA, Jacobson LM, Muehrer RJ, et al. ATR blockade reduces IFN-gamma production in lymphocytes in vivo and in vitro. Kidney Int. 2005;67(6):2134-2142. 